\title{
Correction to: Fit-for-Purpose Quality Control System in Continuous Bioanalysis during Long-Term Pediatric Studies
}

\author{
Mohsin Ali, ${ }^{1}$ Jutta Tins, ${ }^{1}$ and Bjoern B. Burckhardt ${ }^{1,2}$ on behalf of the LENA consortium
}

Correction to: The AAPS Journal (2019) 21: 104.

DOI: https://doi.org/10.1208/s12248-019-0375-1

The LENA collaborator list below was not included in the original article.

LENA collaborator list - update 16.12.2019.

LENA Beneficaries and Principal Investigators.

Prof. Dr. Stephanie Läer, Germany (Coordinator).

Prof. Dr. Jörg Breitkreutz, Germany.

Dr. Ingrid Klingmann, Belgium.

PD Dr. Florian Lagler, Austria.

Prof. Jan de Hoon, Belgium.

Dr. Michiel Dalinghaus, The Netherlands.

Prof. Dr. Milica Bajcetic, Serbia.

Prof. Dr. Saskia de Wildt, The Netherlands.

Anne Keatley Clarke, United Kingdom.

Dr. Johannes Breur, The Netherlands.

Prof. Dr. Christoph Male, Austria.

Dr. Laslo Ablonczy, Hungary.

Prof. Dr. Thomas Mir, Germany.

Prof. Dr. Vladislav Vukomanovic, Serbia.

Prof. Dr. Milan Dukic, Serbia.

Prof. Dr. Ida Jovanovic, Serbia.

Advanced Scientists.

Dr. Bjoern B Burckhardt, Dr. Willi Cawello, Dr. Karl Kleine,

Dr. Angelika Moder, Dr. Emina Obarcanin, Peter Wagner,

Dr. Jennifer Walsh, Dr. Anne van Hecken, Lucie Spatenkova.

\section{PhD Students.}

Mohsin Ali, Dr. Bojana Božić, Dr. Maja Bijelić, Ilja Burdman, Dr. Agnes Ciplea, Muhammed Faisal, Samieh Farahani, Martin Feickert, Tanja Gangnus, Milica Lazic, Dr. Nina Makowski, Fabian Süssenbach, Dr. Marijke van der Meulen, Dr. Saša Popović, Dr. Miro Parezanović, Dr. Nori Smeets, Dr. Vanessa Swoboda.

Clinical investigators.

Dragana Bojanin, Dr. Stefan Đorđević, Jasminka Dragić, Dr. Ann-Kathrin Holle, Dr. Bosiljka Jovičić, Prof Dr. Jovan Košutić, Gordana Kozomara, Haidara Majid, Jadranka Mitrović, Dr. Sanja Ninić, Dr. Miro Parezanovic, Prof Dr. Vojislav Parezanovic, Dr. Andrija Pavlović, Dr.Sergej Prijić, Branislava Rebić, Dr. Igor Stefanović, Dr. Daniel Tordas, Dr. Irena Vulićević.

Study nurses, Technicians, Social workers.

Anke Bartels, Andjelka Čeko, Marissa Herborts, Annelies Hennink, Bosiljka Kosanović, Sanja Kostic, Ljiljana Isailović, Jasmina Maksimovic, Badies Manai, Nada Martinović, Gyöngyi Máté, Miloš Perišić, Jelena Reljić, Regina Pirker Marta Salamomovic, Claudia Schlesner, Jutta Tins, Eva Wissmann.
The online version of the original article can be found at https:// doi.org/10.1208/s12248-019-0375-1

\footnotetext{
${ }^{1}$ Institute of Clinical Pharmacy and Pharmacotherapy, Heinrich Heine University, Universitaetsstr.1, 40225, Dusseldorf, Germany.

${ }^{2}$ To whom correspondence should be addressed. (e-mail: bjoern.burckhardt@hhu.de)
} 